\title{
CONSTITUINTES QUÍMICOS DAS FOLHAS DE Senna spectabilis (DC) IRWIN \& BARNEBY var. excelsa (Schrad.) IRWIN \& BARNEBY
}

\author{
Fábio de Oliveira Silva, Írvila Ricarte de Oliveira e Maria Goretti de Vasconcelos Silva* \\ Departamento de Química Orgânica e Inorgânica, Departamento de Química Analítica e Físico-Química, Universidade Federal \\ do Ceará, Campus do Pici, 60455-970 Fortaleza - CE, Brasil \\ Raimundo Braz-Filho \\ Universidade Estadual do Norte Fluminense, Av. Alberto Lamego, 2000, 28013-600 Campos dos Goytacazes - RJ, Brasil
}

Recebido em 31/12/09; aceito em 15/6/10; publicado na web em 22/9/10

\begin{abstract}
Chemical COMPOUNDS OF LEAVES FROM SENNA SPECTABILIS (DC) IRWIN \& BARNEBY var. excelsa (SCHRAD.) IRWIN \& BARNEBY. From leaves of Senna spectabilis var. excelsa were isolated caffeine, the triterpenes lupeol, $\alpha$-amyrin, $\beta$-amyrin, cycloeucalenol, friedelin and ursolic, oleanolic and betulinic acids, besides the steroids sitosterol and stigmasterol and their respective glucosides. The structures of these compounds were established by spectroscopic analysis including two-dimensional NMR methods and comparison with published spectral data. This paper deals with the first report of these compounds in S. spectabilis var. excelsa.
\end{abstract}

Keywords: Senna spectabilis var. excelsa; triterpene; caffeine.

\section{INTRODUÇÃO}

No Brasil ocorrem 200 gêneros e 1.500 espécies da família Fabaceae. Presente na maioria dos ecossistemas brasileiros, é relatada como a família botânica mais bem representada na caatinga, com 293 espécies em 77 gêneros, constituindo aproximadamente um terço de todos os vegetais deste bioma. ${ }^{1,2}$

O gênero Senna Mill. pertence à tribo Cassieae Bronn, subtribo Cassinae Irwin \& Barneby, juntamente com o gênero Cassia, são fontes importantes de substâncias com grande diversidade estrutural na família Fabaceae. Espécies de Senna são utilizadas tradicionalmente como laxantes e purgativas (Senna alexandrina Mill, syn. Cassia angustifolia Vahl, Senna acutifolia Delile) e como corantes (Senna cernua (Balbis) I. \& B., Senna multijuga (L. C. Rich.) I. \& B. ${ }^{3}$ Atividade laxativa comparável ao padrão bisacodil (princípio ativo da Lacto-purga ${ }^{\circledR}$ ) e anti-inflamatória similar ao diclofenaco de sódio foi observada para o extrato das folhas de Senna macranthera. ${ }^{4}$ Outras diversas atividades relevantes como antimicrobiana, analgésica, antiparasitária, inseticida, antitumoral e hepatoprotetora são comprovadas para várias espécies de Senna. ${ }^{5}$

Espécimes de Senna spectabilis são empregadas no Brasil principalmente no paisagismo, devido à grande beleza das suas flores amarelas, sendo uma espécie com grande potencial para recomposição de áreas degradadas. S. spectabilis ocorre em duas variedades distintas principalmente pelo porte: Senna spectabilis (DC) Irwin \& Barneby var. spectabilis (Cassia spectabilis DC.), (Cassia carnaval), árvore de grande porte (até $17 \mathrm{~m}$ ) e Senna spectabilis (DC) Irwin \& Barneby var. excelsa (Schrad.) Irwin \& Barneby (Cassia excelsa Schrad), de pequeno porte (até $6 \mathrm{~m}$ ) conhecida como cássia-do-nordeste. ${ }^{6}$

Até o presente, o alcaloide piperidínico conhecido por cassina ${ }^{7}$ isolado há mais de 40 anos, é o único registro de estudo químico de Senna spectabilis var. excelsa (Schrad.).

O estudo químico das folhas de S. spectabilis var. excelsa (Schrad) levou ao isolamento e identificação de oito triterpenos pentacíclicos: quatro na forma pura (ácido betulínico, lupeol, cicloeucalenol, friedelina) e quatro como misturas ( $\alpha$-amirina, $\beta$-amirina, ácido ursólico e oleanólico). Apesar de serem substâncias conhecidas, este é o

\footnotetext{
*e-mail: mgvsilva@ufc.br
}

primeiro relato dessa classe química na espécie Senna spectabilis var excelsa. Também foram identificadas duas misturas de esteroides (estigmasterol e $\beta$-sitosterol) na forma livre e glicosilada, além de um alcaloide (cafeína) ainda inédito no gênero Senna.

\section{RESULTADOS E DISCUSSÃO}

O extrato etanólico das folhas de S. spectabilis var. excelsa (Schrad) através de técnicas cromatográficas levou ao isolamento dos triterpenos pentacíclicos ácido betulínico (1), lupeol (2), $\alpha$-amirina e $\beta$-amirina (3 e 4), da fração não alcaloídica (FNA) $(m=13,5 \mathrm{~g})$. A identificação foi possível após a análise dos dados espectrais de RMN ${ }^{1} \mathrm{H}, \mathrm{RMN}{ }^{13} \mathrm{C}$ e DEPT 135 e comparação com dados da literatura. A comparação dos dados obtidos com a literatura permitiu identificar o composto 1 como um triterpeno pentacíclico de esqueleto lupano denominado ácido betulínico. ${ }^{8,9}$

A substância (2) apresentou sinais de $\mathrm{RMN}{ }^{1} \mathrm{H}$ em $\delta_{\mathrm{H}} 4,57$ e 4,69 referentes a hidrogênios vinílicos de dupla terminal e em $\delta_{\mathrm{H}} 3,19$ de hidrogênio carbinólico indicando a presença da $\beta$-configuração além dos sinais em $\delta_{\mathrm{H}} 0,76$ a 1,68 referentes às metilas. O sinal de RMN ${ }^{13} \mathrm{C}$ em $\delta_{\mathrm{C}} 76,8(\mathrm{CH})$, além das absorções em $\delta_{\mathrm{C}} 151,1(\mathrm{C})$ e $\delta_{\mathrm{C}} 109,2$ $\left(\mathrm{CH}_{2}\right)$ caracterizam um triterpeno com esqueleto lup-20 (29)-eno e por comparação dos dados obtidos com a literatura foi possível identificar (2) como lupeol (lup-20(29)-en-3ß-ol). ${ }^{9,10}$

O tratamento cromatográfico da fração diclorometânica levou ao isolamento e identificação de (5), (6) (7) e (8). A substância (5) apresentou-se como um sólido branco amorfo, que após a análise se observaram de $\mathrm{RMN}{ }^{1} \mathrm{H}, \mathrm{RMN}{ }^{13} \mathrm{C}$ e DEPT $135^{\circ}$, se observou sinais em: $\delta_{\mathrm{H}} 4,72(\mathrm{~s})$ e $\delta_{\mathrm{H}} 4,66(\mathrm{~d}, J=1,0)$ característicos de hidrogênios de dupla terminal; em $\delta_{\mathrm{H}} 0,15(\mathrm{~d}, J=4,0)$ e $\delta_{\mathrm{H}} 0,39(\mathrm{~d}, J=4,0)$ referentes a hidrogênios metilênicos; em $\delta_{\mathrm{H}} 1,44(\mathrm{~s})$ e $\delta_{\mathrm{H}} 1,98(\mathrm{~m})$ também referentes a hidrogênios metilênicos; em $\delta_{\mathrm{H}} 3,21(\mathrm{~m})$ característico de hidrogênio ligado a carbono oxigenado (carbono carbinólico); foi possível observar também em $\delta_{\mathrm{H}} 2,24(\mathrm{~m})$ absorção de hidrogênio metínico e em $\delta_{\mathrm{H}} 0,90(\mathrm{~s}), \delta_{\mathrm{H}} 0,98(\mathrm{~s}), \delta_{\mathrm{H}} 0,99(\mathrm{~d}, J=7,1), \delta_{\mathrm{H}} 1,02$ (d, $J=6,8), \delta_{\mathrm{H}} 1,03$ (d, $\left.J=6,8\right)$ absorções de hidrogênios metílicos. A análise dos dados obtidos em comparação com dados da literatura permitiu identificar (5), como um triterpeno de esqueleto cicloartano denominado 4 $\beta$-demetil-24-metilen-cicloartanol (cicloeucalenol), já 

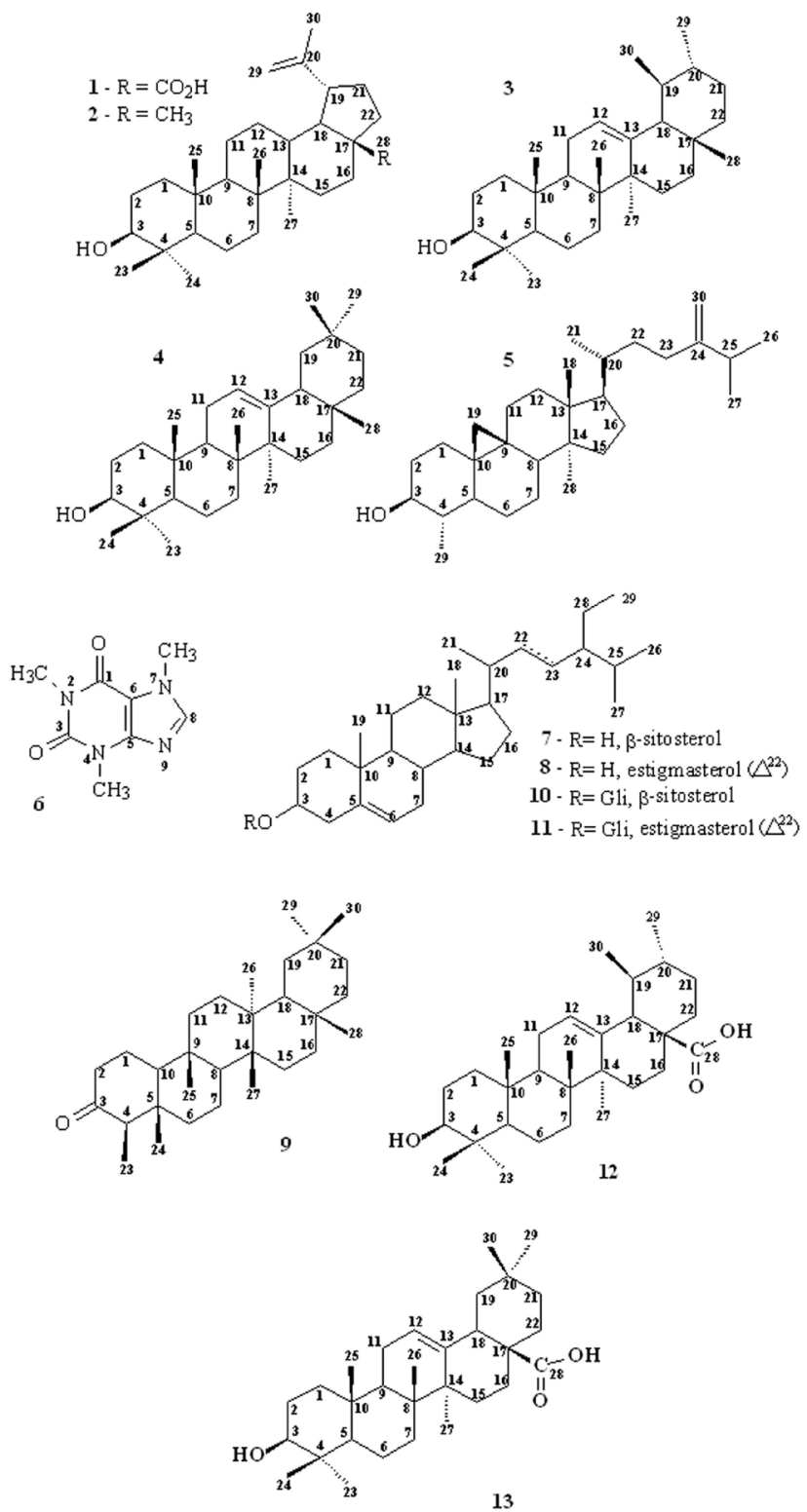

Figura 1. Representação das estruturas das substâncias isoladas das folhas de Senna spectabilis var. excelsa

isolado de várias espécies de plantas e dos moluscos Calyptogena soyoae e Bathymodiolus septemdierum. ${ }^{11-14}$

Para o composto (6), solúvel em clorofórmio, o espectro de RMN ${ }^{1} \mathrm{H}$, apresentou sinal em $\delta_{\mathrm{H}} 7,51(\mathrm{~s})$ para hidrogênio aromático e em $\delta_{\mathrm{H}} 3,99(\mathrm{~s}), \delta_{\mathrm{H}} 3,59(\mathrm{~s})$ e $\delta_{\mathrm{H}} 3,41(\mathrm{~s})$ caracterizados como hidrogênios metílicos. A análise dos dados de $\mathrm{RMN}{ }^{13} \mathrm{C}$ possibilitou observar oito linhas espectrais para átomos de carbono, em $\delta_{\mathrm{C}} 155,54$ e $\delta_{\mathrm{C}} 151,83$, para carbonos carbonílicos; em $\delta_{\mathrm{C}} 148,90$ e $\delta_{\mathrm{C}} 107,83$ referentes a carbonos não hidrogenados e três absorções de carbonos metílicos em $\delta_{\mathrm{C}} 33,88 ; \delta_{\mathrm{C}} 30,03$ e $\delta_{\mathrm{C}} 28,18$. A análise do espectro de massas de alta resolução apresentou os picos $m / z 195$ e 217 [(M+H) e $(\mathrm{M}+\mathrm{Na})]$. A análise dos dados obtidos em comparação com dados da literatura e com amostra autêntica, permitiu identificar (6) como a mais conhecida das metilxantinas, a cafeína ${ }^{15}$ cuja presença foi detectada por CCD também nos extratos de folhas, caule e raízes da planta. Apesar do registro do uso popular, principalmente no Nordeste do Brasil, de espécies do gênero Senna no preparo de uma bebida semelhante ao café ${ }^{16}$ não se encontrou nenhuma referência da presença de cafeína em outras espécies do gênero.
A fração diclorometânica F10-14, após ser submetida à cromatografia gravitacional em coluna, forneceu as substâncias (9), (10), (11), (12) e (13). A análise dos dados de $\mathrm{RMN}{ }^{1} \mathrm{H}$, e de $\mathrm{RMN}{ }^{13} \mathrm{C}$, em comparação com dados da literatura, possibilitou determinar estruturalmente (9) como friedelan-3-ona (friedelina). ${ }^{10,17,18}$

As estruturas das misturas de $\alpha$-amirina (3) e $\beta$-amirina (4); $\beta$-sitosterol (7) e estigmasterol (8) na forma livre; $\beta$-sitosterol (10) e estigmasterol (11) na forma glicosilada; ácido ursólico (12) e oleanólico (13) foram identificadas usando-se padrões em CCDA, análise dos espectros de $\mathrm{RMN}{ }^{1} \mathrm{H}$ e ${ }^{13} \mathrm{C}$, DEPT- $135^{\circ}$ e comparação com dados da literatura. A literatura relata que misturas destas classes são frequentemente tidas como substâncias puras, sendo sua separação considerada muito difícil. ${ }^{8,10}$

O estudo realizado com $S$. spectabilis var. excelsa contribui para o conhecimento da química do gênero Senna e da flora nordestina. Todas as substâncias isoladas e identificadas neste trabalho não foram descritas previamente na espécie Senna spectabilis var excelsa.

\section{PARTE EXPERIMENTAL}

\section{Procedimentos experimentais gerais}

Na determinação dos pontos de fusão (p.f.) das substâncias isoladas usou-se um aparelho de microdeterminação Microquímica, provido de placa aquecedora modelo MQAPF-301. A determinação foi feita a uma velocidade de aquecimento de $1{ }^{\circ} \mathrm{C} / \mathrm{min}$, e não houve correção. O processo cromatográfico de separação foi majoritariamente de adsorção. Nos experimentos cromatográficos de adsorção em coluna (CC) foi utilizado gel de sílica (0,063-0,200 mm, 70-230 mesh, Vetec), enquanto para os experimentos de cromatografia em camada delgada analítica (CCDA) foram utilizadas cromatoplacas de gel de sílica, com indicador de fluorescência na faixa de 254 $\eta \mathrm{m}$ (Merck). As substâncias foram reveladas sob luz ultravioleta (254 e 366 ๆm) e/ou pela aspersão com solução de vanilina/ácido perclórico/EtOH, seguida de aquecimento ou, ainda, pela exposição a vapores de iodo.

Os espectros de RMN foram produzidos em espectrômetros Bruker, modelos Avance DPX-300 e DRX-500, operando na frequência do hidrogênio a 300 e $500 \mathrm{MHz}$, e na frequência do carbono a 75 e $125 \mathrm{MHz}$, respectivamente. Os espectros de massas de baixa resolução foram obtidos em cromatógrafo em fase gasosa acoplado a espectrômetro de massas (CG-EM), modelo QP5050A da Shimadzu, operando a 70 eV. O espectro de massas de alta resolução foi adquirido usando um espectrômetro de massas mod. LCMS-IT-TOF (225-07100-34) - Shimadzu, equipado com fonte de ionização por.

\section{Material vegetal}

As folhas de Senna spectabilis var. excelsa foram coletadas em agosto de 2003 no município de Boa Viagem - CE, de árvores adultas e sua exsicata encontra-se depositada no Herbário Prisco Bezerra sob o número 33.013, no Departamento de Biologia da Universidade Federal do Ceará - UFC, Fortaleza, Ceará, Brasil.

\section{Extração e isolamento}

O material botânico foi triturado, seco e extraído exaustivamente com etanol $(95 \%)$ a frio e concentrado em evaporador rotativo, obtendo-se 39,0 g. O extrato das folhas foi então submetido à técnica de extração seletiva de alcaloides, ${ }^{19}$ obtendo-se assim uma fração alcaloídica (FA) (m=15,8 g), que está sendo estudada e uma não alcaloídica (FNA) (m=13,5 g). A fração não alcaloídica foi submetida à coluna cromatográfica (CC) em gel de sílica, eluída com hexano, 
diclorometano, acetato de etila e metanol, na forma isocrática e/ou usando gradiente de eluição. A subfração diclorometânica F04-09, foi então submetida a novo processo cromatográfico, fornecendo a fração F10-23C, que após análise por CCDA e determinação do ponto de fusão resultou no isolamento do ácido $3 \beta$-hidroxi-lup-20(29)-en28-óico (ácido betulínico, 1, 12,0 mg, p.f. $285,2{ }^{\circ} \mathrm{C}$ lit. ${ }^{9} 285,0{ }^{\circ} \mathrm{C}$ ), lup-20(29)-en-3ß-ol (lupeol, 2, 8,0 mg, p.f. $246,0^{\circ} \mathrm{C}$, lit. ${ }^{9} 212,0{ }^{\circ} \mathrm{C}$ )

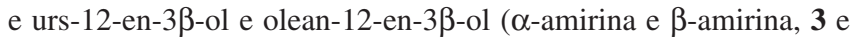
4, $90,0 \mathrm{mg}$, p.f. $178,4^{\circ} \mathrm{C}$ ).

O tratamento cromatográfico da subfração F48-53E, oriunda da fração diclorometânica F04-09, forneceu um sólido branco amorfo, que após análise em CCDA e determinação do seu ponto de fusão foi identificada como $4 \beta$-demetil 24-metilencicloartanol (cicloeucalenol, 5, 26,0 mg, p.f. $143,0{ }^{\circ} \mathrm{C}$, lit. ${ }^{12} 139,0{ }^{\circ} \mathrm{C}$ ). $\mathrm{O}$ tratamento por coluna cromatográfica e posterior análise por CCD da fração FDF04-09 resultou na obtenção das subfrações FDF48-53E e FDF28-53G. A subfração FDF48-53E forneceu um sólido branco amorfo (17 mg) com faixa de fusão $149,3-150,1{ }^{\circ} \mathrm{C}$ identificado como cafeína (6). Amostras dos extratos etanólicos de folhas, caule e raízes foram submetidas à comparação através de CCDA com o composto 6 e padrão autêntico de cafeína (Rf = 0,62; fase móvel: diclorometano/metanol 95:5), revelando por similaridade de Rfs que, em todas as partes da planta testadas, a cafeína estava presente. A partir do tratamento cromatográfico de FDF28-53G obteve-se um sólido na forma de agulhas incolores (25 mg), solúvel em clorofórmio e faixa de fusão $140-142{ }^{\circ} \mathrm{C}$, que após análise dos dados foi identificado com a mistura dos esteroides $\beta$-sitosterol e estigmasterol (7) e (8).

A investigação da fração diclorometânica F10-14, ao ser submetida à CC, CCDA e determinação do ponto de fusão das substâncias isoladas, permitiu identificar: friedelan-3-ona (friedelina, 9, 28,0 mg, p.f. $258,7^{\circ} \mathrm{C}$, lit. ${ }^{17} 249,0^{\circ} \mathrm{C}$ ) e ácido $3 \beta$-hidroxi-urs-12-en-28-óico (ácido ursólico) e $3 \beta$-hidroxi-olean-12-en-28-óico (ácido oleanólico), 12 e $13,9,0 \mathrm{mg}$, p.f. $269,1^{\circ} \mathrm{C}$ ).

\section{AGRADECIMENTOS}

Ao CNPq, à CAPES e FUNCAP, pelas bolsas concedidas e apoio financeiro no desenvolvimento dessa pesquisa, ao Centro Nordestino de Aplicação e Uso da Ressonância Magnética Nuclear (CENAUREMN), pela obtenção dos espectros de RMN e ao Laboratório de Espectrometria de Massas do Nordeste (LEMANOR).

\section{REFERÊNCIAS}

1. Córdula, E.; Dissertação de Mestrado, Universidade Federal de Pernambuco, Brasil, 2008.

2. Melo-Pinna, G. F. A.; Neiva, M. S. M.; Barbosa, D. C. A.; Rev. Bras. Bot. 1999, 375 .

3. Sá, I. M.; Valle, L. S.; Almeida, G. S.; Rev. Bras. Bio. 2007, 5, 276.

4. Nogueira, L. G.; Dissertação de Mestrado, Universidade Federal de Juiz de Fora, Brasil, 2009.

5. Viegas, C. J.; Rezende, A.; Silva, D. H. S.; Castro-Gambôa, I.; Bolzani, V. S.; Quim. Nova 2006, 29, 1279; Takkis, K.; Sild, S.; Maran, U.; QSAR Comb. Sci. 2009, 28, 829; Macedo E. M. S.; Wiggers, H. J.; Silva, M. G. V.; Montanari, C. A.; Braz Filho, R.; J. Braz. Chem. Soc. 2009, $20,947$.

6. Irwin, H. S.; Barneby, R. C.; The American Cassiinae: A synoptical Revision of Leguminoseae tribe Cassia subtribe Cassinae in the new word, The New York Botanical Garden: New York, 1982.

7. Highet, R. J.; J. Org. Chem. 1964, 29, 471.

8. Falcão, D. Q.; Dissertação de Mestrado, Universidade Federal do Rio de Janeiro, Brasil, 2008.

9. Silva, V. C.; Alves, A. N.; Santana, A.; Carvalho, M. G.; Silva, S. L. C.; Schripsema, J.; Quim. Nova 2006, 29, 1184.

10. Carvalho, M. G.; Velandia, J. R.; Oliveira, L. F.; Bezerra, F. B.; Quim. Nova 1998, 21, 740.

11. Kongkathip, N.; Dhumma-upakorn, P.; Kongkathip, B.; Chawananoraset, K.; Sangchomkaeo, P.; Hatthakitpanichakul, S.; J. Ethnopharmacol. 2002, 83, 95 .

12. Paula, J. R., Vieira, I. J. C.; Silva, M. F. G. F.; Fernandes, J. B.; Vieira, P. C.; Pinheiro, A. L.; Vilela, E. F.; Phytochemistry 1997, 44, 1449.

13. Haba, H.; Lavaud, C.; Harkat, H.; Magid, A. A.; Marcourt, L.; Benkhaled, M.; Phytochemistry 2007, 68, 1255.

14. Hawai, S.; Takada, W.; Tsuchida, S,; Kado, R.; Kimura, J.; Fish. Sci. 2007; 73, 902 .

15. Montrucchio, D. P.; Miguel, G.; Miguel, M. D.; Monache, F. D.; Carvalho, J. L. S.; Visão acadêmica 2005, 6, 1518

16. Lombardo, M.; Kiyota, S.; Kaneko, T.M.; Rev. Ciênc. Farm. Básica Apl. $30, \mathbf{2 0 0 9}, 1$

17. Costa, F. J.; Bandeira, P. N.; Albuquerque, M. R. J.; Pessoa, O. D. L.; Silveira, E.; Braz-Filho, R.; Quim. Nova 2008, 31, 1691.

18. David, J. M.; Santos, F. A.; Guedes, M. L. S.; David, J. P.; Quim. Nova 2003, 26, 484.

19. Matos, F.J.A.; Introdução à Fitoquímica Experimental, $3^{\mathrm{a}}$. Ed. EUFC: Fortaleza, 2009. 